\title{
Short communication Short-term presurgical treatment for all?
}

Mitch Dowsett ${ }^{1}$, Janine Salter ${ }^{1}$, Margaret Hills ${ }^{1}$, Simone Detre ${ }^{1}$, Roger A'Hern ${ }^{2}$ and lan E Smith ${ }^{3}$

\author{
1Department of Academic Biochemistry, Royal Marsden Hospital, London, SW3 6JJ, UK \\ 2Institute of Cancer Research, Clinical Trials and Statistics Unit, Sutton, Surrey, SM2 5PT, UK \\ ${ }^{3}$ Breast Unit, Royal Marsden Hospital, London, SW3 6JJ, UK
}

Corresponding author: Mitch Dowsett, mitch@icr.ac.uk

Published: 20 December 2007

This article is online at http://breast-cancer-research.com/content/9/S2/S13

(c) 2007 BioMed Central Ltd

\section{Introduction}

There are two types of presurgical treatment of primary breast cancer [1]: one in which clinical downstaging is the goal and end-point, and one that is shorter in duration and does not involve delay to normal surgical scheduling. The latter, socalled 'window of opportunity' studies of about 2 weeks' duration, are the focus of this article.

Short-term presurgical studies require biomarkers for their assessments of pharmacological effectiveness. These same biomarkers may be intermediate markers of eventual clinical efficacy if the treatment were to be continued. Given that increases or decreases in tumour growth require changes in the balance between cell multiplication and loss, markers of the processes have become important biomarkers for presurgical treatment. There is now substantial data to support their validity as markers of therapeutic benefit for both chemotherapy and endocrine therapy.

\section{Proliferation}

The most widely used measurement of proliferation is immunohistochemical assessment of Ki67. We recently reviewed its biology and importance in breast cancer [2]. Other measures of proliferation used in breast cancer include flow cytometric assessment of S-phase, mitotic index (a component of pathologic grade) and thymidylate synthase. None of these markers provides a measure of the proliferative rate because they are static, snapshot-in-time measures. The incorporation of bromodeoxyuridine (BrDU) allows a measurement of proliferative rate, but this is a rarely performed measurement because of its complexity and some safety concerns. The pre-eminence of Ki67 is largely because of the relative simplicity of its measurement. The most widely used antibody of recent years has been MIB1.

\section{Cell death}

Cell death in relation to medical therapy appears largely to be as a result of apoptotic programmed cell death. This may be measured by morphologic criteria using standard
Breast Cancer Research 2007, 9(Suppl 2):S13 (doi:10.1186/bcr1811)

microscopic techniques, but it is more often measured in vivo by application of the TUNEL (TdT-mediated dUTP-biotin nick end labeling) methodology that links biotinylated nucleotides to the enormous number of DNA strand ends created during late-stage apoptosis. The immunohistochemical staining of components of the execution machinery of apoptosis, such as activated caspase 3 , have been assessed as surrogates for apoptosis itself with variable success $[3,4]$. The role of necrosis in the response to treatment of human breast cancer appears to be limited, although this probably accounts for a significant proportion of the tumour dynamics seen in rapidly regressing xenograft models. Autophagy may also contribute to cell death in response to some treatments, but this process also acts as a survival mechanism during the early response to metabolic stress [5], making it difficult to assess the importance of autophagy to tumour cell loss.

\section{Assay precision}

For widespread applicability it is necessary to have well defined assay precision. We assessed the precision of making measurements of Ki67 and apoptosis by taking two cores with 14-gauge needles from the same tumour at the same time without intervening treatment [6]. The mean error was 33\% (standard deviation [SD] 16\%) for Ki67 and 38\% (SD 22\%) for apoptosis. These data provided statistical power for our early studies in which these markers were primary end-points. Capturing the data from the placebo arm of some of those studies subsequently allowed us to expand the precision data. In a similar study using fine needle aspirates rather than cores, it became clear that the larger proportional but smaller absolute differences seen at low values of the markers created an error relationship that was not completely linear [7].

\section{Relationship between proliferation and apoptosis}

There is a significant direct correlation between Ki67 levels and apoptosis in breast cancer [8]. This probably explains the counterintuitive observation that when apoptosis is assessed 
as a univariate it is associated with poor prognosis, but when both proliferation and apoptosis are included in the model apoptosis no longer exhibits a significant association with clinical outcome [8]. Assessment of apoptosis as well as proliferation does not appear to improve prediction of outcome above proliferation alone. However, its assessment as a changing parameter in relation to treatment response is of substantial importance, particularly in terms of chemotherapy.

\section{Chemotherapy}

Higher proliferation at baseline is among several biomarkers that exhibit an inverse association between response to neoadjuvant chemotherapy and long-term outcome (for a review see [2]). This may be confounding when extrapolating biomarker data in the neoadjuvant setting to clinical outcome in the adjuvant setting.

Apoptosis can be seen to increase significantly in most, but not all, patients during the first 24 hours of chemotherapy [9]. Initial data suggested that this might predict benefit from neoadjuvant chemotherapy [10], but further work indicated that the relationship is poor at best [11]. This may be due to variable pharmacokinetics and pharmacodynamics over this early period. Proliferation is also reduced modestly in this early period, but the changes are not closely related to clinical response. Assessments at 21 days have been more consistent in indicating a relationship between decreased proliferation and eventual clinical response to chemotherapy $[2,7,10]$.

We recently assessed the relationship of Ki67 pretreatment and at surgery with recurrence-free survival (RFS) in patients receiving anthracyclin-based chemotherapy [12]. Before treatment, we found the expected relationship between high Ki67 and poor outcome $(P=0.0007)$. In the excision specimen the relationship was stronger $(P<0.0001)$; median RFS was about 18 months for patients with Ki67 in the highest tertile, as compared with more than 7 years for the other two tertiles. The data suggest that although patients with high proliferation pretreatment have a good chance of a pathological complete remission, if they do not achieve this then their outcome is likely to be very poor.

Despite these positive and potentially clinically helpful data, for short-term presurgical studies of chemotherapy to become widely used, more data on long-term significance after 21 days or less are needed along with evidence that this would usefully alter clinical practice.

\section{Endocrine therapy}

Several studies have assessed changes in proliferation and/or apoptosis as biological efficacy measures of endocrine treatments. The neoadjuvant IMPACT (Immediate Preoperative Anastrozole Tamoxifen or Combined with Tamoxifen) trial randomly assigned postmenopausal patients with primary breast cancer to anastrozole, tamoxifen, or the combination of anastrozole and tamoxifen [13,14]. This randomization was identical to that in the ATAC (Arimidex, Tamoxifen Alone or Combination) adjuvant trial [15]. The reduction in Ki67 with anastrozole was also greater than that with tamoxifen or the combination both at 2 weeks and at 12 weeks, thus predicting the RFS differences in ATAC. This provokes the question of whether ATAC would have persisted with the combination arm had data from IMPACT been available before its initiation. Exclusion of this arm would have incurred major savings at many levels. Our recent report that anastrozole plus gefitinib was no more effective than anastrozole alone in suppressing $\mathrm{Ki} 67$ in the presurgical setting is discouraging for the development of that combination for early breast cancer treatment [16].

Unexpected decreases in apoptosis have been observed after treatment with aromatase inhibitors and tamoxifen $[14,17]$. These may be due to the close relationship between apoptosis and proliferation discussed above.

Unlike Ki67, clinical response in IMPACT did not exhibit the differences between the three treatment arms that were seen in RFS in ATAC. This may be due to the imprecision in measuring clinical response but there may be other, more fundamental reasons. After 2 weeks of anastrozole or tamoxifen, 52 out of 56 and 46 out of 54 patients, respectively, exhibited decreased Ki67. Thus, almost all patients appeared to have some antiproliferative benefit, but this varied markedly between patients. In particular, some patients with high Ki67 levels before treatment exhibited a modest fall by 2 weeks. Such falls would not be expected to be associated with the regression of a rapidly growing tumour, but if considered in the adjuvant setting such changes might be associated with improved RFS. Thus, it is rational that there might be a closer relationship between change in proliferation in the neoadjuvant setting and benefit from adjuvant therapy than from response in the neoadjuvant setting itself.

Given that baseline Ki67 is prognostic and the evidence above suggests that a 2-week change in Ki67 is predictive of benefit, it is rational that the absolute value of Ki67 at 2 weeks might integrate the intrinsic prognostic importance of Ki67 at baseline and the predictive value of change; it would therefore be expected to relate more closely to longterm outcome with a given treatment than baseline Ki67. This hypothesis was confirmed in the IMPACT trial, where the 2-week value of Ki67 had a better association with RFS than the pretreatment value [18]. To translate these findings into routine clinical practice requires the relationship between 2-week Ki67 and RFS to be characterized more fully. This should be accomplished by the POETIC (Peri-Operative Endocrine Therapy for Individualized Care) trial, a large clinical study of aromatase inhibitor therapy, or no such therapy, before surgical resection, which will also provide 
tissue to allow detailed study of the molecular changes resulting from oestrogen deprivation; this may allow improved insight into the mechanisms that underpin benefit from endocrine therapy.

\section{Conclusion}

Treatment-induced, short-term presurgical changes in proliferation and apoptosis have an established role in evaluation of new drugs and show promise for prediction of benefit from existing treatments of primary breast cancer. Research is ongoing to determine whether they may merit assessment in most patients.

\section{Acknowledgement}

This article has been published as part of Breast Cancer Research Volume 9 Supplement 2, 2007: Controversies in Breast Cancer. The full contents of the supplement are available online at http://breastcancer-research.com/supplements/9/S2.

\section{References}

1. Dowsett $\mathrm{M}$ : Preoperative models to evaluate endocrine strategies for breast cancer. Clin Cancer Res 2003, 9:502S-510S.

2. Urruticoechea A, Smith IE, Dowsett M: Proliferation marker Ki67 in early breast cancer. J Clin Oncol 2005, 23:7212-7220.

3. Parton M, Krajewski S, Smith I, Krajewska M, Archer C, Naito M, Ahern R, Reed J, Dowsett M: Coordinate expression of apoptosis-associated proteins in human breast cancer before and during chemotherapy. Clin Cancer Res 2002, 8:2100-2108.

4. Hadjiloucas I, Gilmore AP, Bundred NJ, Streuli CH: Assessment of apoptosis in human breast tissue using an antibody against the active form of caspase 3: relation to tumour histopathological characteristics. Br J Cancer 2001, 85:1522-1526.

5. Gozuacik D, Kimchi A: Autophagy and cell death. Curr Top Dev Biol 2007, 78:217-245.

6. Ellis PA, Smith IE, Detre S, Burton SA, Salter J, A'Hern R, Walsh G, Johnston SR, Dowsett M: Reduced apoptosis and proliferation and increased $\mathrm{Bcl}-2$ in residual breast cancer following preoperative chemotherapy. Breast Cancer Res Treat 1998, 48:107-116.

7. Assersohn L, Salter J, Powles TJ, A'hern R, Makris A, Gregory RK, Chang J, Dowsett M: Studies of the potential utility of Ki67 as a predictive molecular marker of clinical response in primary breast cancer. Breast Cancer Res Treat 2003, 82:113-123.

8. Lipponen P, Aaltomaa S, Kosma VM, Syrjanen K: Apoptosis in breast cancer as related to histopathological characteristics and prognosis. Eur J Cancer 1994, 30A:2068-2073.

9. Ellis PA, Smith IE, McCarthy K, Detre S, Salter J, Dowsett M: Preoperative chemotherapy induces apoptosis in early breast cancer. Lancet 1997, 349:849.

10. Chang J, Ormerod M, Powles TJ, Allred DC, Ashley SE, Dowsett $\mathrm{M}$ : Apoptosis and proliferation as predictors of chemotherapy response in patients with breast carcinoma. Cancer 2000, 89: 2145-2152.

11. Archer CD, Parton M, Smith IE, Ellis PA, Salter J, Ashley S, Gui G, Sacks N, Ebbs SR, Allum W, et al.: Early changes in apoptosis and proliferation following primary chemotherapy for breast cancer. Br J Cancer 2003, 89:1035-1041.

12. Jones RL, Salter J, Nerurkar A, Parton M, A'Hern $R$, Smith IE, Dowsett M: Prognostic (Px) significance of Ki67 before and after neoadjuvant chemotherapy (CT) in early breast cancer. $J$ Clin Oncol 2007, 25:573.

13. Dowsett M, Smith IE, Ebbs SR, Dixon JM, Skene A, Griffith C, Boeddinghaus I, Salter J, Detre S, Hills M, et al.; IMPACT Trialists: Short-term changes in Ki-67 during neoadjuvant treatment of primary breast cancer with anastrozole or tamoxifen alone or combined correlate with recurrence-free survival. Clin Cancer Res 2005, 11:951s-958s.

14. Smith IE, Dowsett M, Ebbs SR, Dixon JM, Skene A, Blohmer JU, Ashley SE, Francis S, Boeddinghaus I, Walsh G; IMPACT Trialists Group: Neoadjuvant treatment of postmenopausal breast cancer with anastrozole, tamoxifen, or both in combination: the Immediate Preoperative Anastrozole, Tamoxifen, or Combined with Tamoxifen (IMPACT) multicenter double-blind randomized trial. J Clin Oncol 2005, 23:5108-5116.

15. Baum M, Budzar AU, Cuzick J, Forbes J, Houghton JH, Klijn JG, Sahmoud T; ATAC Trialists' Group: Anastrozole alone or in combination with tamoxifen versus tamoxifen alone for adjuvant treatment of postmenopausal women with early breast cancer: first results of the ATAC randomised trial. Lancet 2002, 359:2131-2139.

16. Smith IE, Walsh G, Skene A, Llombart A, Mayordomo JI, Detre S, Salter J, Clark E, Magill P, Dowsett M: A phase II placebo-controlled trial of neoadjuvant anastrozole alone or with gefitinib in early breast cancer. J Clin Oncol 2007, 25:3816-3822.

17. Harper-Wynne CL, Sacks NP, Shenton K, MacNeill FA, Sauven $P$, Laidlaw IJ, Rayter Z, Miall S, Howes A, Salter J, et al.: Comparison of the systemic and intratumoural effects of tamoxifen and the aromatase inhibitor vorozole in postmenopausal patients with primary breast cancer. J Clin Oncol 2002, 20:1026-1035.

18. Dowsett M, Smith IE, Ebbs SR, Dixon JM, Skene A, A'Hern R, Salter J, Detre S, Hills M, Walsh G; IMPACT Trialists Group: Prognostic value of Ki67 expression after short-term presurgical endocrine therapy for primary breast cancer. J Nat/ Cancer Inst 2007, 99:167-170. 